\title{
A Cross-Layer Scheme for Handover in 802.16e Network with F-HMIPv6 Mobility
}

\author{
Yi ZHENG, Yong ZHANG, Yinglei TENG, Mei SONG \\ Beijing University of Posts and Telecommunications, Beijing, China \\ Email: \{zhengyibupt, bjzhangyong, lilytengtt\}@gmail.com; Songm@bupt.edu.cn
}

\begin{abstract}
IEEE802.16e is the major global cellular wireless standard that enables low-cost mobile Internet application. However, existing handover process system still has latency affects time-sensitive applications. In this paper, the handover procedures of 802.16e and Fast Handover for Hierarchical MIPv6 (F-HMIPv6) are reconstructed to achieve a better transmission performance. The concept of cross layer design is adopted to refine the existing handover procedure specified in 802.16e MAC layer and F-HMIPv6. More specifically, layer2 and layer3 signaling messages for handover are analyzed and combined/interleaved to optimize the handover performance. Extensive simulations show that the proposed scheme in this paper is superior to the other scheme proposed by IETF.
\end{abstract}

Keywords: handover, cross layer, 802.16e, F-HMIPv6

\section{Introduction}

Recently, wireless access technologies have been evolving for diverse capabilities and services. The Third Generation Partnership Project (3GPP) has been defining Universal Terrestrial Radio Access (UTRA) for $3 \mathrm{G}$ radio access, as well as the optimization of the network architecture with HSxPA [1]. The CDMA2000 mobile communication system also has been evolved into $1 \mathrm{xEV}$-Dx for high speed data services. As one of wireless access technologies, Mobile WiMAX was successfully adopted by ITU as one of the IMT-2000 technologies in November 2007. Since then mobile WiMAX (IP-OFDMA) has officially become a major global cellular wireless standard along with 3GPP UMTS/HSPA and 3GPP2 CDMA/ EVDO [2].

Mobile WiMAX is a fast growing broadband access technology that enables low-cost mobile Internet applications, and realizes the convergence of mobile and fixed broadband access in a single air interface and network architecture. The IEEE802.16e provides high bandwidth, low-cost, scalable solutions that extend services from backbone networks to wireless users. Because of a larger coverage area, portability and mobility have become significant issues for providing high quality application, as it is crucial to minimize handover latency and maintain session continuity. The IEEE $802.16 \mathrm{e}$ standard only defines a frame work in MAC layer (L2) without considering upper layer handover performance. But from the IP based service point of view, simply reducing the L2 latency does not adequately reduce the overall handover latency. The whole handover procedure shall not include L2 only but also the IP layer. So in order to improve the IP layer (L3) handover performance, a number of standards of MIPv6 are proposed by IETF. Fast Handovers for Mobile
IPv6 (FMIPv6) [3], aim to reduce the handover latency by configuring new IP addresses before entering the new subnet. Hierarchical MIPv6 mobility management (HMIPv6) [4] introduces a hierarchy of mobile agents to reduce the registration latency and the possibility of an outdated care-of address. FMIPv6 and HMIPv6 can also be used together as suggested in [5] to reduce the latency related to Movement Detection and CoA configuration/ Verification and cut down the signaling overhead and delay concerned with Binding Update (BU). Fast Handover for Hierarchical MIPv6 (F-HMIPv6) [5] also introduces the Mobility Anchor Point (MAP) to provide a better solution for micro mobility.

In order to provide seamless services during handover, in this paper, we study the process that shall be performed in L2 and L3 and the related message of 802.16e and FHMIPv6 to propose a cross layer handoff scheme. In order to speed up the total handover process, we also use the proposed scheme in [6] to optimize the 802.16e network entry procedure and reduce the L2 handover delay. This paper is organized as follows. In Section 2 we briefly introduce the relevant protocols and related proposals. In Section 3 we present our cross-layer handover scheme, while Section 4 validates its performance. We finally conclude the paper in Section 5.

\section{Background and Related Works}

\subsection{Handover Process of 802.16e}

Figure 1 shows the handover process of 802.16e. The $802.16 \mathrm{e}$ handover $(\mathrm{HO})$ procedure includes several phases, namely, network topology acquisition and advertisement, target BS scanning procedure, $\mathrm{HO}$ decision and initiation, and network re-entry [7]. We provide details about these 
stages, explaining what is their role in the overall MAClayer HO latency.

In network topology acquisition stage, a BS periodically broadcasts the system information of the neighboring BSs using Neighbour Advertisement message. The Serving BS may unicast MOB_NBR-ADV message based on the cell types of neighbor cells, in order to achieve overhead reduction and facilitate scanning priority for MS. Then MS may use any unavailable intervals assigned by the serving $\mathrm{BS}$ to perform scanning. The BS or MS may prioritize the neighbor BSs to be scanned based on various metrics, such as cell type, loading, RSSI and location. MS measures the selected scanning candidate BSs and reports the measurement result back to the serving BS by using Neighbour Advertisement message.

The handover algorithm is a network-controlled, MSassisted handover. Although handover procedure may be initiated by either MS or BS, the final $\mathrm{HO}$ decision and target $\mathrm{BS}(\mathrm{s})$ selection are performed by the serving $\mathrm{BS}$ or the network. MS executes the $\mathrm{HO}$ as directed by the $\mathrm{BS}$ or cancels the HO procedure through HO cancellation message. The network re-entry procedure with the target BS may be optimized by target BS possession of MS information obtained from serving BS over the backbone network. MS may also maintain communication with serving BS while performing network re-entry at target BS as directed by serving BS.

\subsection{Fast Handover for Hierarchical MIPv6 (F-HMIPv6)}

The handover process of 802.16e mainly deals with layer2 hop-by-hop connection issues. However, fast Handover for Hierarchical MIPv6 (F-HMIPv6) protocol takes the advantage of FMIPv6 and HMIPv6, which is the most popular layer3 protocol. The HMIPv6 introduces the Mobility Anchor Point (MAP) to reduce the signaling overhead and delay concerned with Binding Update for micro mobility. Therefore HMIPv6 still needs a further handover enhancement to support the real-time applications. Currently FMIPv6 is the typical protocol to reduce the handover latency. Then F-HMIPv6 integrates these two protocols and provides a scheme for effective integration. Figure 2 illustrates the generic procedures for $\mathrm{F}$ HMIPv6 operations.

Based on L2 handover anticipation, the mobile node (MN) sends RtSolPr message to MAP. The RtSolPr should include information about the link layer address or identifier of the concerned New Access Router (NAR). In response to the RtSolPr message, the MAP sends the PrRtAdv message contain information about New on-link Care of Address (NLCoA) to the MN. At this time, MAP

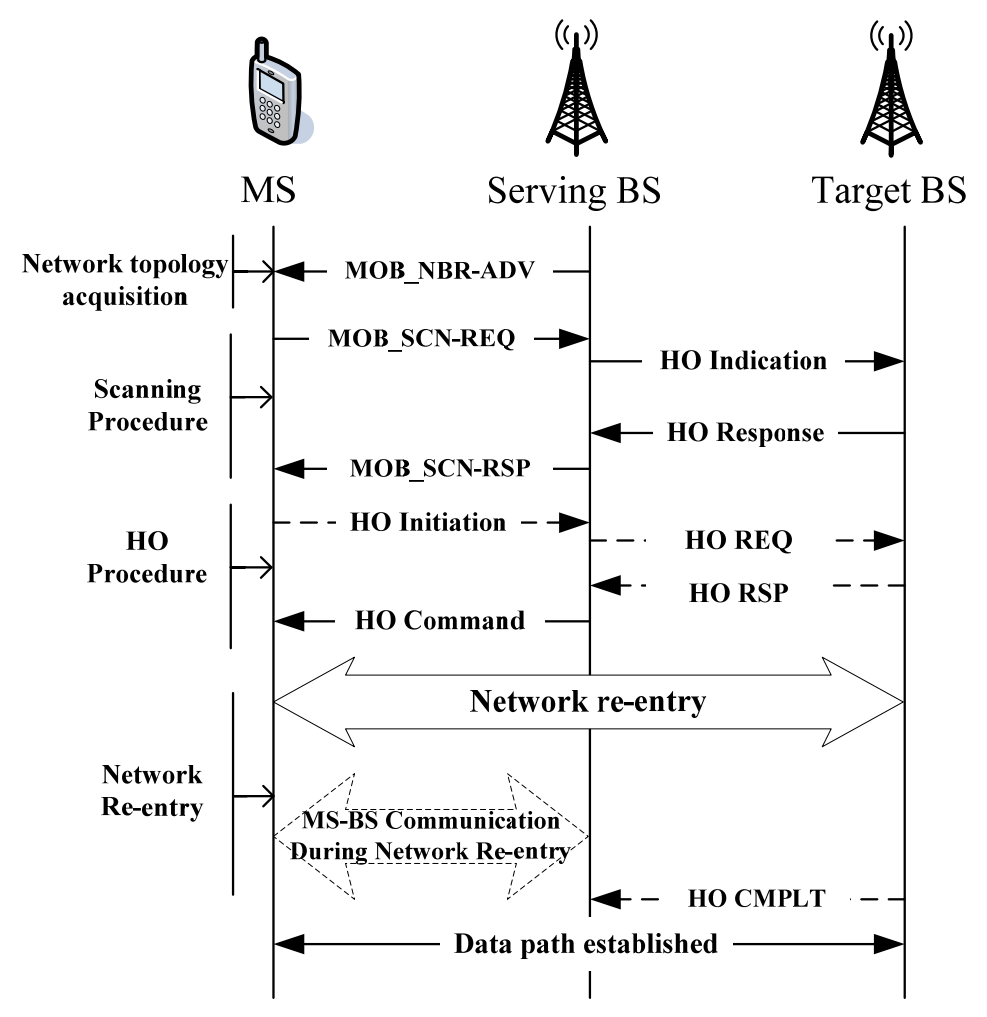

Figure 1. A general flow for HO 


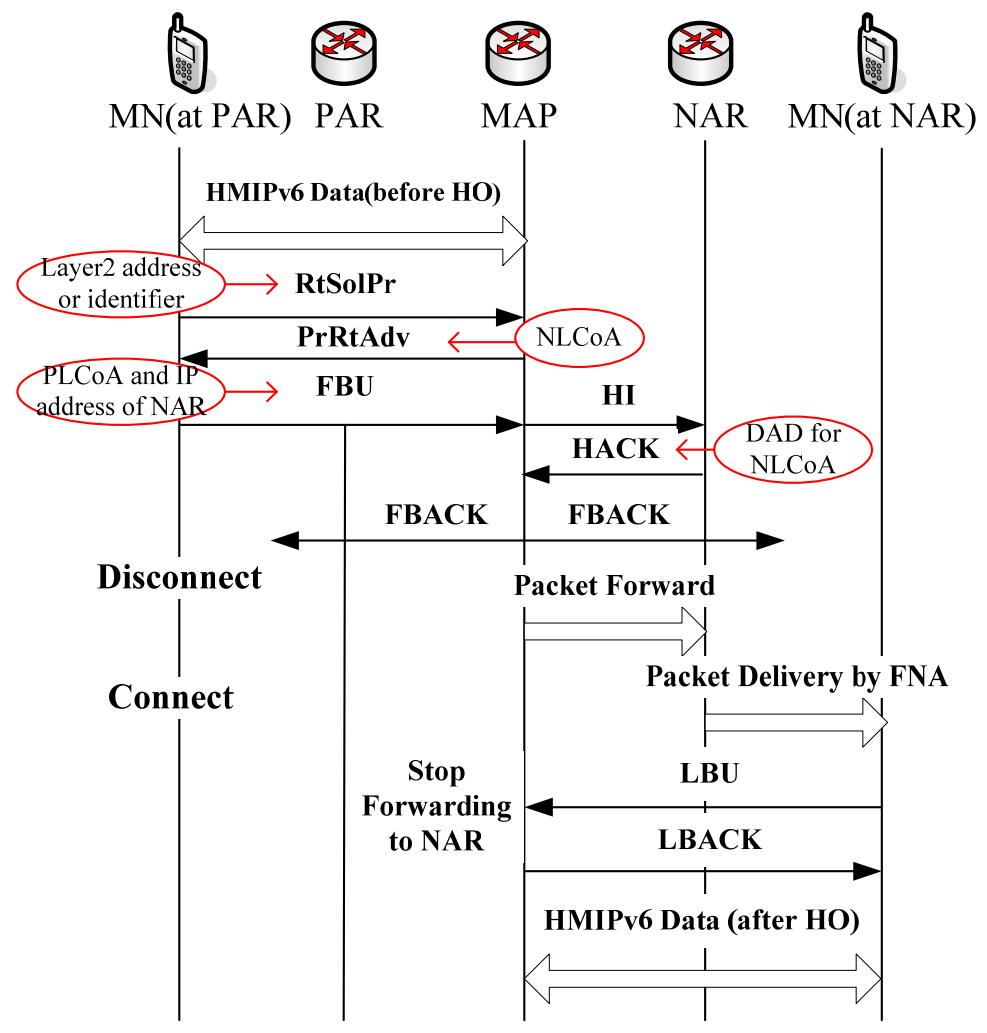

Figure 2. Procedures of F-HMIPv6

has already known the network prefix and link layer address of the associated NAR. Subsequently, MN will update MAP by sending Fast Binding Update (FBU) message which contains previous on-link CoA (PLCoA) and IP address of the NAR. After receiving the FBU message from MN, the MAP will send a Handover Initiate (HI) message to the NAR so as to establish a bi-directional tunnel. If Handover Acknowledgement (HACK) message from NAR indicates the validity of new NLCoA by Duplicate Address Detection (DAD) procedure, the bi-directional tunnel between MAP and NAR will set up. All data packets are intercepted by MAP and delivered over this tunnel. The MN sends Fast Neighbor Advertisement (FNA) messages to NAR, when it detects that it is moved in the link layer, and the NAR delivers the buffered data packets to the MN over NLCoA. When the MAP receives the new Local Binding Update with NLCoA from the MN, it will stop the packet forwarding to NAR and then clear the tunnel established for fast handover. When MN sends Local Binding Update (LBU) to MAP, MAP will respond with Local Binding ACK (LBACK), and forward packet directly to the MN (at NAR).

\section{Proposed Cross-Layer Handover Scheme}

In this section, we propose our cross layer handover scheme (CLHS). The CLHS achieves seamless handover by exploiting the L2 handover indicators and designing an efficient interleaving scheme of the 802.16e and the F-HMIPv6 handover procedures. The basic idea of CLHS is as follows: 1) integrate the correlated messages of 802.16e and F-HMIPv6. 2) reorder/combine L2 and L3 signaling messages and minimize the required control flow. Thus we can get shorter handover latency and higher throughput.

Figure 3 shows network architecture of CLFS in 802.16e.To achieve hierarchical handover, we propose a MAP in ASN (Access Service Network) Gateway. The MAP acts as an aggregation mobile anchor residing in ASN Gateway and connecting with Access Router (AR). If MN moves between subnets in the same MAP domain, it should be in intra-MAP handover status. If MN moves from one MAP domain to another, it should be in inter-MAP handover status. If MN moves between subnets in the same AR domain, it should be in L2 handover status.

Identical with HMIPv6 scheme, a MN has two CoAs, on-link CoA (LCoA) and Regional CoA (RCoA). When MN enters a new MAP domain, firstly it performs the HMIPv6 registrations procedures with HA and MAP. Then MN will bind its LCoA with an address on the MAP's RCoA. If the MN changes its current address within a local MAP domain (LCoA), such as from AR1 to AR2, it only needs to register the new address with the 


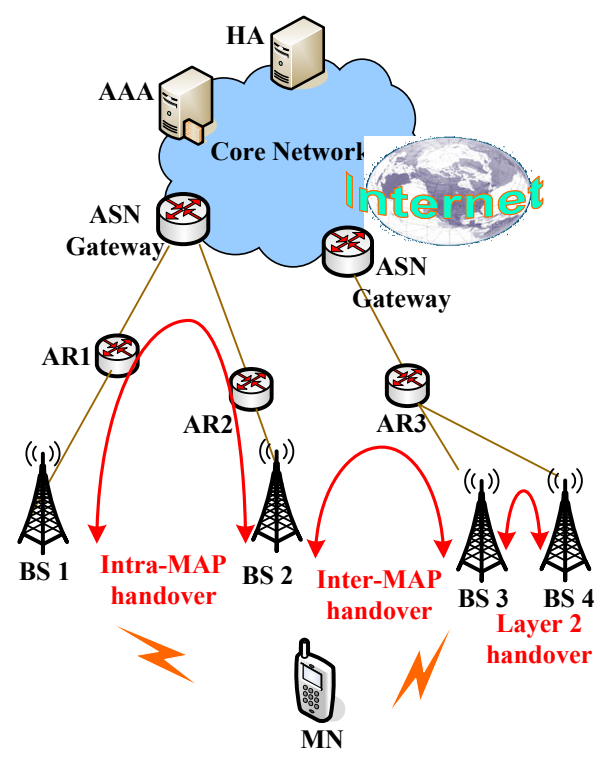

Figure 3. Network architecture of CLFS in 802.16e

MAP, following the Local Binding Update procedures of HMIPv6. As long as MN moves from AR2 to AR3 in the picture, the Regional CoA (RCoA) needs to be registered with correspondent nodes and the HA. When MN moves from BS3 to BS4, it only needs the MAC Layer handover.

In Figure 4, we design F-HMIPv6 handover information integrate with $802.16 \mathrm{e}$ and make some modifications. The "MOB_NBR_ADV" message is periodically sent by BS and its function is similar to the "PrRtAdv" message in F-HMIPv6. So, these two periodical advertisement messages can be combined together. We can deliver the
L3 information of target network which MN moves to in MOB_NBR_ADV message, and RtSol/RtAdv messages can be omitted. By combining 802.16e with F-HMIPv6, and employing the former's new BS discovery ability with the RtSol/RtAdv messages, MN movement could be detected. In addition to modifying these two messages, we can make a little modification and combine the message of FBU in layer 3 and the message of MOB HO IND in layer 2. It is indicate that L2'handover when MS sends MOB_HO_IND message; And FBU message is to inform MAP for the initiation of L3' handover. Therefore, it's reasonable to send MOB_HO_IND together with FBU.

In the first place, S-BS shall broadcast a MOB_NBR -ADV including L3 information of RtSol/RtAdv to MN periodically. If the $\mathrm{MN}$ discovers a new neighbor $\mathrm{BS}$ in this message, it may perform scanning for the T-BS. When the MN decides to change links based on its policy such as the degrading signal strength or increasing packet loss rate, it will initiate handover by sending a MOB_MSHO-REQ to the BS and will receive a MOB BSHO-RSP from the $\mathrm{BS}$ as a response. Alternatively, the BS may initiate handover by sending a MOB_BSHO -REQ to the MN. Then MN sends MOB_HO_IND together with FBU to S-BS, and S-BS will forward FBU message to MAP. After that, messages of HI and HACK occur between the MAP and NAR to implement DAD and establish a bi-directional tunnel. As soon as the tunnel is set up, MAP sends FBACK messages over previous LCoA (PLCoA) and new LCoA (NLCoA), and intercepting packets destined for the MN to NAR over this tunnel. After switching links, the MN synchronizes

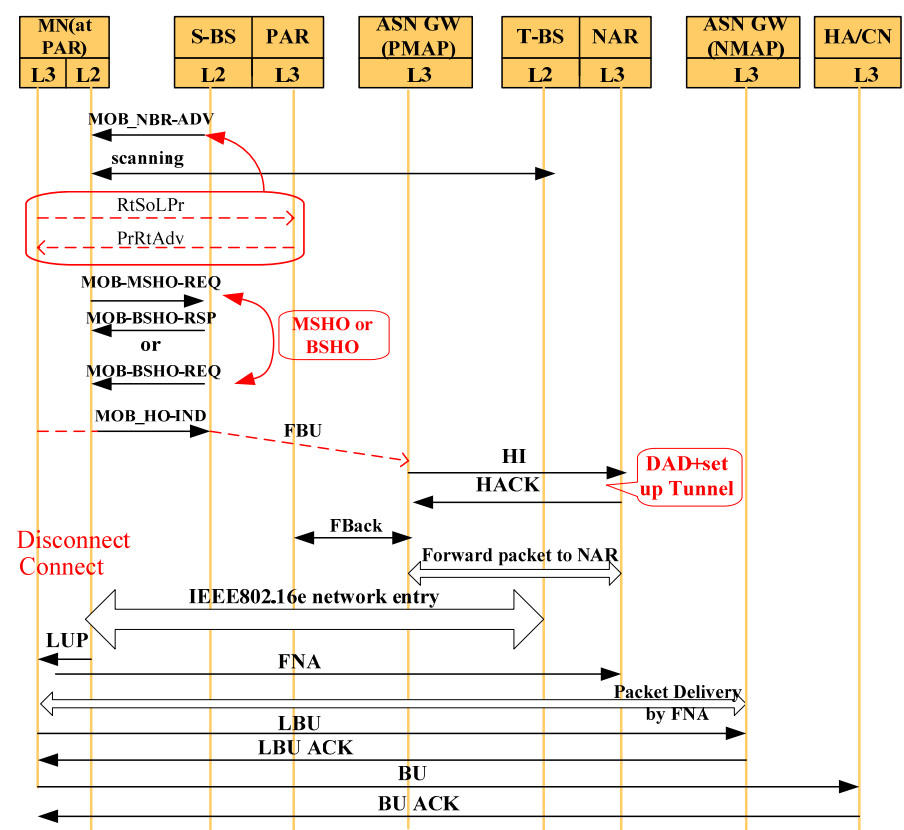

Figure 4. CLFS mechanism for 802.16e 
with the target BS and performs the 802.16e network entry procedure. In this process, $\mathrm{MN}$ will acquaint of $\mathrm{NCoA}$ by Fast_DL_MAP_IE message [7]. Once the network entry procedure is completed, the L2 signals L3 with a LINK_UP (LUP) which report MN establish L2 connection with T-BS. In this stage, MN issues an FNA to NAR by using NLCoA as a source IP address. When the NAR receives the FNA from $\mathrm{MN}$, it delivers the buffered packets to the MN through the tunnel. Then MN sends LBU message to NMAP to bind the NRCoA and NLCoA, and NMAP replies LBACK. In the end, NMAP delivers the buffered packets to $\mathrm{MN}$ through the tunnel. Thus, the whole handover operation is completed.

The above is the general flow of CLFS mechanism, in practice, different cases need different processes. When $\mathrm{MN}$ from one AR to another with micro mobility, there will be only needs to register the new address with the MAP, following the Local Binding Update procedures of HMIPv6. Therefore, we don't detail here.

\section{Performance Analysis}

In this section, we provide a performance analysis for the concept described in Section 3. The performance evaluation here provided is performed by means of simulations carried out with Matlab.

\subsection{Analytical Models}

In this paper, we consider that the handover latency starts with MN send MOB_HO_IND together with FBU, and completes with $\mathrm{MN}$ can normally communicate with $\mathrm{CN}$. To analyze the performance of proposed scheme, we define some parameters in the Table 1 as following:

If we assume $\mathrm{T}_{\mathrm{L} 2}$ is the $\mathrm{L} 2$ handover delay, the conventional $\mathrm{L} 2$ handover delay is

$$
T_{L 2(c)}=T_{\text {sync }}+T_{\text {cont_ resol }}+T_{\text {rng }}+T_{\text {auth }}+T_{\text {reg }}+T_{\text {frame }}
$$

In Formula (1), $\mathrm{T}_{\text {sync }}$ can be done within 2 frames. $\mathrm{T}_{\text {cont resol }}$ typically needs minimum of 6 frames roundtrip delay plus a random handling duration. $\mathrm{T}_{\text {auth }}$ needs 2

Table 1. Notations of performance parameters

\begin{tabular}{ll}
\hline Symbol & \multicolumn{1}{c}{ Descriptions } \\
\hline $\mathrm{T}_{\mathrm{a} \_\mathrm{b}}$ & Delay between node a and node $\mathrm{b} ;$ \\
$\mathrm{T}_{\text {frame }}$ & Frame duration of IEEE802.16e PHY \\
$\mathrm{T}_{\text {DAD }}$ & Average delay of the DAD procedure; \\
$\mathrm{T}_{\text {tunnel a_b }}$ & Latency for Tunneled packets; \\
$\mathrm{T}_{\text {hop }}$ & Delay of each hop in a wired backbone network \\
$\mathrm{N}_{\mathrm{a} \_\mathrm{b}}$ & Number of hops between node a and node b; \\
$\mathrm{T}_{\text {sync }}$ & Average time required to synchronize with new downlink; \\
$\mathrm{T}_{\text {cont resol }}$ & Average time required for contention resolution procedure; \\
$\mathrm{T}_{\text {rng }}$ & Average time required for ranging process during HO; \\
$\mathrm{T}_{\text {auth }}$ & Average time required for re-authorization during HO; \\
$\mathrm{T}_{\text {reg }}$ & average time required for re-registration; \\
\hline
\end{tabular}

frames plus a handling duration of about $100 \mathrm{~ms}$. $\mathrm{T}_{\text {reg }}$ always needs 2 frames plus a handling duration of about

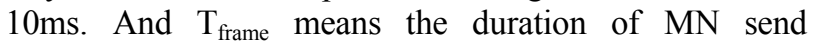
MOB_HO_IND together with FBU message which takes 1 frame.

We also give the L2 and L3 total handover delay $\mathrm{T}_{\text {total }}$ of conventional FMIPv6 in [8] as follow:

$$
\begin{aligned}
& T_{\text {total (FMIIPV } 6)}=2 T_{P_{\text {AR }} \text { NAR }}+T_{D_{\text {AAD }}}+T_{\text {tunnelPAR_NAR }_{-}}+T_{L 2(c)} \\
& +2 T_{M N_{-} H A}+2 T_{M N_{-} C N}+3 T_{M N_{-} A R} \\
& =2\left(N_{P A R_{-} N A R}+N_{\text {NAR }_{-} H A}+N_{\text {NAR }_{-} C N}\right) \times T_{\text {hop }} \\
& +T_{D A D}+T_{\text {tumnelPAR_NAR }}+T_{L 2(c)}+7 T_{M N_{-} A R}
\end{aligned}
$$

From the Formula (1), we may see that it takes a long time for handover procedures in conventional L2 scheme. That's mainly because, in case of network entry produce, MS fails to receive Fast RNG_IE and conducts contention based ranging. The MS performs random backoff and sends CDMA codes instead of RNG-REQ message on the link. Furthermore, the MS should perform re-authorization and re-registration processes. If we use the proposed scheme in [8], the downlink packet could be transmitted just after synchronization to the new downlink and total L2 handover delay is cut down. So we can get optimized L2 handover delay as follow:

$$
T_{\text {L2(o) }}=T_{\text {sync }}+T_{\text {frame }}
$$

The total handover latency of the CLHSmechanism is calculated as follow:

$$
\begin{aligned}
& T_{\text {total (CLFS) }}=\max \left(T_{L 2(o)}, T_{D A D}+2 T_{P_{M A P_{-} N A R}}\right. \\
& +T_{P_{M A P_{-} P A R}}+T_{\text {tunnelMAP }-A R} \text { ) } \\
& +T_{M N_{-} N A R}+2 T_{M N_{-} N M A P}+2 T_{M N_{-} H A} \\
& =\max \left(T_{L 2(o)}, T_{D A D}+2\left(N_{P_{A R_{-} N A R}}+N_{M A P_{-} A R}-1\right)\right. \\
& \left.\times T_{\text {hop }}+T_{\text {tunnelMAP_AR }}\right)+T_{M N_{-} \text {NAR }} \\
& +2 T_{M N_{-} N M A P}+2 T_{M N_{-} H A}
\end{aligned}
$$

Then we list the delay period and packet disruption time with CLFS based 802.16e in Figure 5. Apart from handover disruption and delay period, we also note how the time point of trigger influences these operations, as well as the time point at which $\mathrm{CN}$ can send packet directly to $\mathrm{MN}$.

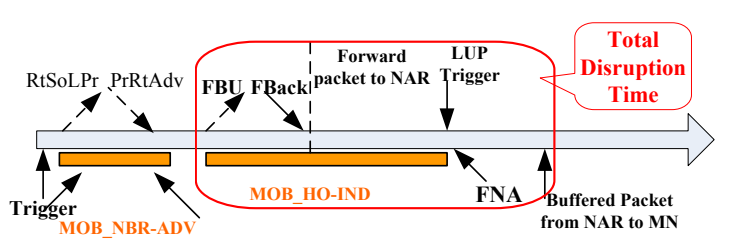

Figure 5. Handover disruption time and delay period 
Related to Figure 5, we can calculate the disruption time of CLFS and conventional way are derived below.

$$
\begin{gathered}
D_{C L F H}=T_{L 2(o)}+T_{M N_{-} N A R} \\
D_{\text {FMIPv } 6}=T_{L 2(c)}+T_{M N_{-} N A R}
\end{gathered}
$$

\subsection{Simulation Results}

We now present the results based on previous analysis. To evaluate the schemes, we assume the parameters as the below table. The NAR and PAR are assumed the same distance from the HA. We assume $T_{\text {hop }}=T_{\text {frame }}+0.5 \mathrm{~ms}$, and the DAD time is chosen from Poisson distribution: [400ms, 600ms].

The simulation results are divided into two parts. The first part is mainly related to the handover latency and the second part is related to the service disruption time for the proposed scheme and the conventional scheme.

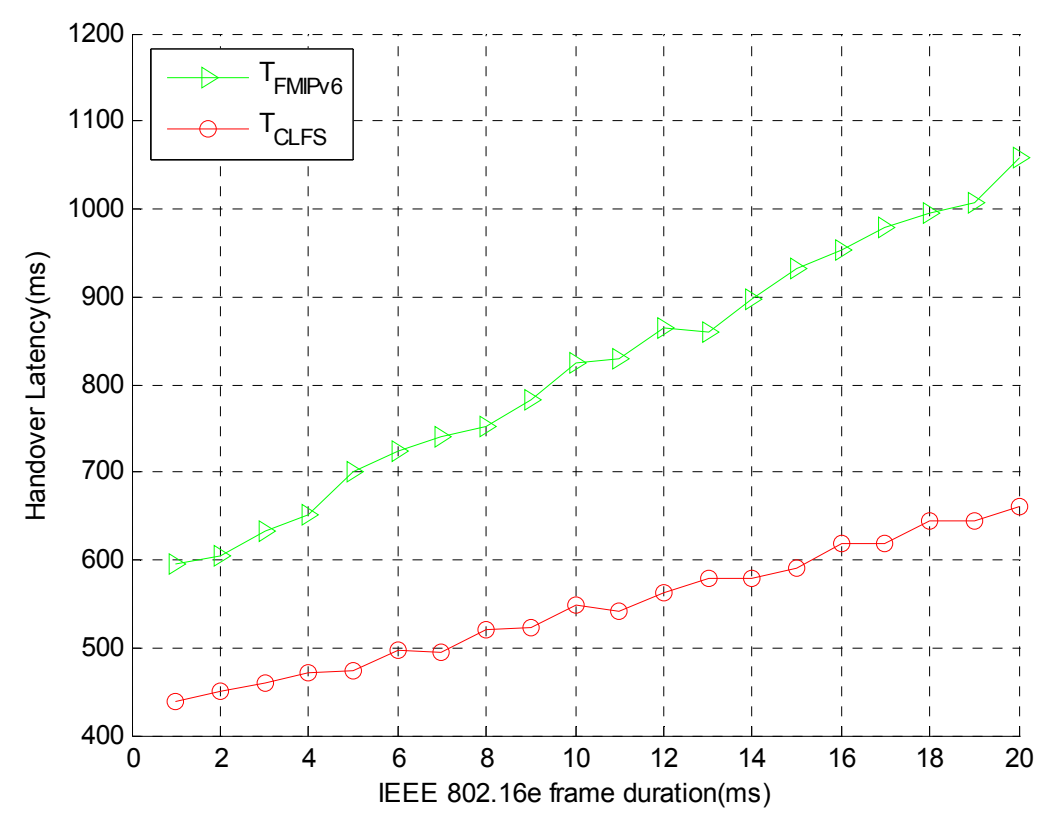

Figure 6. Handover latency for different frame durations

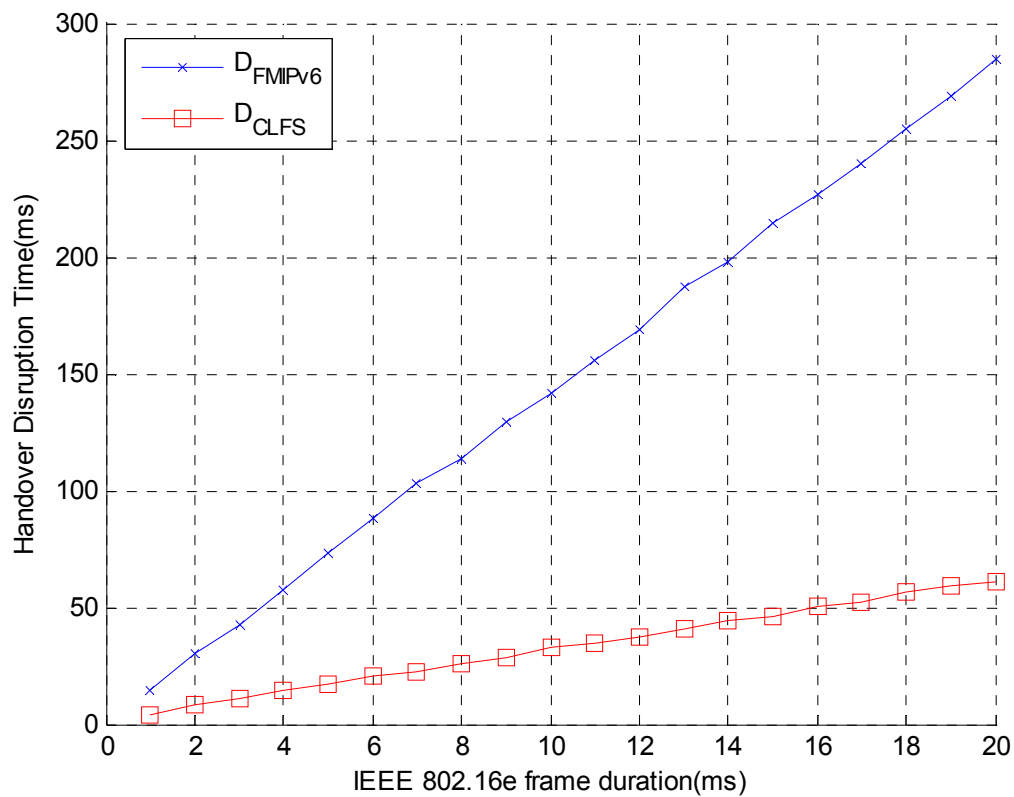

Figure 7. Handover disruption time for different frame durations 
Figure 6 compares the handover delay of the proposed CLHS and the conventional scheme. It's noted that the delay time is mainly reduced in CLHS mechanism. The reason is that our scheme needs less number of messages and L2 and L3 signaling messages are properly arranged for parallelism. When performing handover, the CLHS mechanism can greatly reduce the handover latency and optimize the performance.

In Figure 7, we can find the same situation as Figure 6. When the frame duration increases, the FMIPv6 disruption time grows sharply, whereas our scheme only has a slight increase. It's mainly because that no more registration procedure and authorization procedure are needed in this optimized scheme. Therefore, optimized L2 handover scheme reduce the disruption time.

Table 2. Simulation parameter setting

\begin{tabular}{cc||cc}
\hline Parameter & Value & Parameter & Value \\
\hline $\mathrm{N}_{\text {PAR_NAR }}$ & 6 & $\mathrm{~T}_{\text {NAR_CN }}$ & 10 \\
$\mathrm{~N}_{\text {NAR_HA }}$ & 8 & $\mathrm{~T}_{\text {tunnel a_b }}$ & $10 \mathrm{msec}$ \\
\hline
\end{tabular}

\section{Conclusions}

In this paper, we have proposed a cross-layer optimization with F-HMIP for WiMAX. Our CLHS mechanism reduces the number of signaling messages by combining L2 and L3 messages and parallelizing L2 and L3 signaling messages. In our CLHS mechanism, we use an optimized L2 handover scheme with a Fast_DL_MAP_IE message to enhance the performance and reduce network entry messages. In addition, we compare our scheme with the previous scheme through exhaustive simulations. However, the selection mechanism of an appropriate BS is not within our consideration for simulation simplification. So future research will extend the concept of cross layer to cover the complete handover procedure and optimize the utilization of network resources.

\section{REFERENCES}

[1] BEHESHTI B D. Software implementation and performance analysis of the LTE physical layer blocks on a next generation baseband processor platform. 2008 IEEE Long Island, May 2008, $1-1$.

[2] WANG F, GHOSH A, SANKARAN C, FLEMING P, HSIEH F, BENES S. Mobile WiMAX systems: Performance and evolution. IEEE Communications Magazine, Oct. 2008, 46(10): 41-49.

[3] KOODLI R. Mobile IPv6 fast handovers. IETF RFC5268, June 2008.

[4] SOLIMAN H, CASTELLUCCIA C, MALKI K E, ET AL. Hierarchical mobile IPv6 mobility management (HMIPv6). IETF RFC5380, Oct. 2008.

[5] JUNG H Y. Fast handover for hierarchical MIPv6. IETF Internet-draft, draft-jung-mobopts-fhmipv6-00.txt, 2005.

[6] CHOI S, HWANG G H, KWON T, LIM A R, CHO D H. Fast handover scheme for real-time downlink services in IEEE 802.16e BWA system. VTC 2005-Spring, 3: 2028-2032.

[7] IEEE 802.16e. Amendment 2: Physical and medium access control layers for combined fixed and mobile operation in licensed bands and corrigendum 1. Feb. 28, 2006.

[8] JANG H J. Mobile IPv6 fast handovers over IEEE 802.16e networks. IETF RFC5270, June 2008. 